\title{
Violin varnish induced changes in the vibro-mechanical properties of spruce and maple wood
}

\section{Journal Article}

\section{Author(s):}

Lämmlein, Sarah Louise; Van Damme, Bart; Mannes, David; Schwarze, Francis Willis Matthew Robert; Burgert, Ingo

Publication date:

2020-08

Permanent link:

https://doi.org/10.3929/ethz-b-000438550

Rights / license:

In Copyright - Non-Commercial Use Permitted

Originally published in:

Holzforschung 74(8), https://doi.org/10.1515/hf-2019-0182 
Sarah Louise Lämmlein*, Bart Van Damme, David Mannes, Francis Willis Matthew Robert Schwarze and Ingo Burgert

\section{Violin varnish induced changes in the vibro- mechanical properties of spruce and maple wood}

https://doi.org/10.1515/hf-2019-0182

Received July 11, 2019; accepted November 7, 2019

\begin{abstract}
Violin varnishes are known to alter the longitudinal and radial material properties of violin tonewood. Here, the varnish-induced changes in the vibrational and elastic properties of spruce and maple wood were studied by modal analysis of rectangular plates. This contact-free investigation yields the eigenfrequencies and corresponding mode shapes and modal damping ratios of multiple out-of-plane modes. The longitudinal and radial E-modulus and the in-plane shear modulus were determined by an inverse material property determination process. The influence of traditional European varnish materials and different coating build-ups, comprising alcohol-based as well as oil-based varnishes, was studied during their application and for several months during drying and aging of the multi-layered systems. The evolutions of the changes induced by the different varnishes were rather similar and, except for modal damping, less pronounced for maple than for spruce. For the latter, the longitudinal E-modulus was slightly decreased while the shear modulus and radial E-modulus were increased after 18 months. The strong increases in modal damping ratios shortly after applying the coating systems (up to $150 \%$ ) were reduced over time but remained significant for the time-span studied. However, also small differences between the influence of different coating systems
\end{abstract}

\footnotetext{
*Corresponding author: Sarah Louise Lämmlein, Swiss Federal Laboratories for Materials Science and Technology (Empa), Dübendorf, Switzerland; and Swiss Federal Institute of Technology Zürich (ETH Zürich), Zurich, Switzerland, e-mail: Sarah.Laemmlein@ empa.ch. https://orcid.org/0000-0001-8674-7612

Bart Van Damme: Swiss Federal Laboratories for Materials Science and Technology (Empa), Dübendorf, Switzerland

David Mannes: Paul Scherrer Institute (PSI), Villigen, Switzerland Francis Willis Matthew Robert Schwarze: Swiss Federal Laboratories for Materials Science and Technology (Empa), St. Gallen, Switzerland

Ingo Burgert: Swiss Federal Laboratories for Materials Science and Technology (Empa), Dübendorf, Switzerland; and Swiss Federal Institute of Technology Zürich (ETH Zürich), Zurich, Switzerland
}

and varnish materials on the amplitude of the induced changes became apparent.

Keywords: maple, mechanical properties, modal analysis, non-destructive testing, spruce, violin varnish, wood dynamics

\section{Introduction}

Besides aesthetical rationales and their protective function against humidity changes and wear, it is recognized that violin varnishes influence the vibrational properties of wood (Schelleng 1968; Ono 1993; Minato et al. 1995; Schleske 1998; Obataya et al. 2001; Sedighi Gilani et al. 2016; Lämmlein et al. 2019). The (tacit) knowledge about the varnish procedure of the renowned Cremonese violin makers, however, was not continuously handed down and got lost over the years (Cattani et al. 2013). Therefore, most studies focus on revealing the varnish compositions and the preparation methods used by the famous luthiers (Echard and Lavedrine 2008; Echard et al. 2010; Bucur 2016; Spinella et al. 2017; Fiocco et al. 2019).

The varnish influence on the eigenfrequencies, damping properties and elastic moduli has mostly been studied on wooden strips before and after varnish application. Typically, the strips were excited, either electromagnetically or with an impact hammer, to vibrate in free-free flexural modes. The results, commonly based on the low-frequency modes, mainly show that for Norway spruce wood (Picea abies L.), the longitudinal stiffness was slightly reduced, whereas the radial stiffness and especially the damping properties were (strongly) increased by varnishes (Hutchins 1991; Minato et al. 1995; Schleske 1998; Sedighi Gilani et al. 2016; Lämmlein et al. 2019). Over time, during drying and aging of the coating, the effect of the varnish is further subjected to changes (Schelleng 1968; Holz 1995; Minato et al. 1995; Schleske 1998; Sedighi Gilani et al. 2016). The described changes are visualized in Figure 1. The figure also shows the effects on the torsional properties, which have so far not been described in the literature, but which are reported in this publication. 


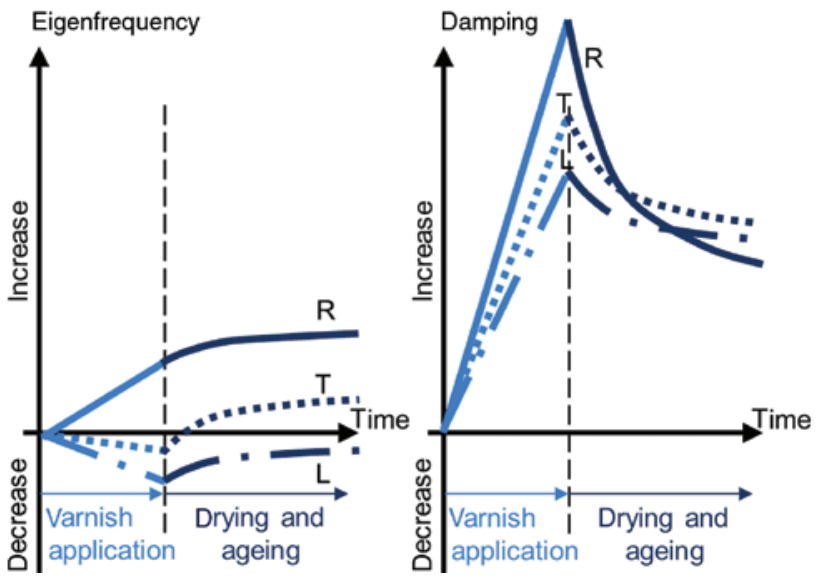

Figure 1: General trends for varnish-induced changes in eigenfrequency (left) and damping ratios (right) for spruce (for the $R$ radial, $\mathrm{L}$ longitudinal and $\mathrm{T}$ torsional vibration modes) compared to the unvarnished state.

The varnish impact is associated with the different elastic constants of tonewood and varnish materials. Wood, as an anisotropic material, has a higher longitudinal E-modulus $\left(\mathrm{E}_{\mathrm{L}}\right)$ and a lower radial E-modulus $\left(\mathrm{E}_{\mathrm{R}}\right)$ than most traditional isotropic varnish materials (Obataya et al. 2001; Simonnet et al. 2002). In addition, varnish materials have a higher density and higher loss factors than spruce and maple (Acer pseudoplatanus L.), the wood species usually used for the construction of the front and back plates of a violin. Moreover, previous studies revealed that the varnish influence depends on the type of varnish applied and on the multi-layer build-up (Hutchins 1991; Schleske 1998, 2002). However, most studies investigated specific varnishes or coating systems without identifying the individual and cumulated contribution of treatment layers.

Besides the importance of $E_{R}$ and $E_{L}$, numerical sensitivity analyses showed an important influence of the in-plane shear modulus $\mathrm{G}_{\mathrm{LR}}$ on violin top and back plates (Rodgers 1988; Viala et al. 2016) and, generally, also of the maple back on the final eigenfrequencies of the entire instrument (Viala et al. 2016). Nonetheless, measurements on maple are very rare and one can find more general comments on the damping properties (Meinel 1957; Eichelberger 2006), eigenfrequency changes (Woo Yang Chung 2000) or a single measurement of the impact of a particular varnish (Haines 1980). The potential influence of varnish on the shear properties of both, spruce and maple, has not been studied to date.

In this publication, the influence of differently combined traditional multi-layer and multi-material coating systems on the vibrational and elastic properties of spruce and maple wood, and the evolution of these changes over time, were investigated. To study this, wideband modal analysis was performed on rectangular spruce and maple plates. Being non-invasive, this approach allows to measure the changes during the varnishing process and in a dynamic manner, as for real instruments. The method yields the eigenfrequencies and corresponding modal damping ratios. The in-plane elastic moduli, i.e. $E_{R}, E_{L}$ and $G_{L R}$, were obtained with an inverse method by minimizing the difference between the eigenfrequencies of a numerical model and the experimental measurements. The applicability of this method has previously been demonstrated not only on simple rectangular plates (Larsson 1997; Pérez Martínez et al. 2011), but also for more complex geometries such as violin soundboards (Viala et al. 2018). Due to the rectangular plate geometry, this approach allows an assessment of the influence on the shear properties and a determination of the anisotropic properties in one measurement on a single sample.

\section{Materials and methods}

Wood and coating materials: Fifty spruce plates of $140 \times 100 \times 2.9 \mathrm{~mm}^{3}(\mathrm{~L} \times \mathrm{R} \times \mathrm{T})$ and 30 maple plates of $95 \times 60 \times 2.6 \mathrm{~mm}^{3}$ $(\mathrm{L} \times \mathrm{R} \times \mathrm{T})$ with planed radial (LR) surfaces were sampled out of quarter-sawn wood blocks. The wood for the specimens was taken from the same trees and in the case of the spruce plates all specimens were twin samples (five rows in the tangential direction each with 10 samples aligned in the longitudinal direction). The mean dimensions, weight and density are summarized in Table 1 . In order to avoid potential superposition of the fundamental eigenmodes [see equations (4)-(6)] and to obtain a significant number of samples, different dimensions for spruce and maple were chosen.

Following the traditional violin varnishing methods, the studied coating systems comprised several layers and different varnish materials. Referring to the Byzantine finishing system as described by Koen Padding (Padding 2005), which is still being used today and allows a systematic analysis of different possible build-ups as encountered on antique and modern instruments, the following coating materials were chosen and applied with a brush:

Table 1: Dimensions and weights of studied samples at $20^{\circ} \mathrm{C}$ and $65 \%$ RH.

\begin{tabular}{lrr}
\hline & Spruce plates $\varnothing(\mathbf{s t d})$ & Maple plates $\varnothing(\mathbf{s t d})$ \\
\hline Mass $(\mathrm{g})$ & $18.49(0.12)$ & $9.77(0.16)$ \\
Length $(\mathrm{mm})$ & $140.02(0.03)$ & $95.12(0.02)$ \\
Width $(\mathrm{mm})$ & $100.20(0.10)$ & $60.44(0.05)$ \\
Thickness $(\mathrm{mm})$ & $2.894(0.011)$ & $2.56(0.02)$ \\
Density $\left(\mathrm{kg} \mathrm{m}^{-3}\right)$ & $455.3(2.7)$ & $665.1(6.2)$ \\
\hline
\end{tabular}

The standard errors of measurement (SEM) are reported in Supplementary material S3. Std, standard deviation. 
- $\quad$ Primer (N), one layer: $4.3 \mathrm{wt} \%$ sodium nitrite $\mathrm{NaNO}_{2}$ dissolved in distilled water

- Sealing (S), one layer: mastic resin dissolved in turpentine (Mastic Varnish from Kremer Pigmente GmbH \& Co. KG, Aichstetten, Germany)

- Grounding (G), one layer: clear oil varnish (OldWood classical amber, OldWood Colours \& Varnishes S.L., Madrid, Spain) and $29 \mathrm{wt} \%$ pumice powder (Pumice Powder 6/0 from Kremer Pigmente GmbH \& Co. KG, Aichstetten, Germany)

- Alcohol varnish (A), four layers: known as “1704 varnish”, based on shellac (Shellac Orange from Kremer Pigmente GmbH \& Co. KG, Aichstetten, Germany), gum elemi (from Hammerl GmbH \& Co. KG, Baiersdorf, Germany), spike oil (Spike-Lavender Oil from Kremer Pigmente GmbH \& Co. KG, Aichstetten, Germany) and ethanol

- Oil varnish (0), four layers: Hammerl standard quality oil varnish golden brown.

The varnish application was performed by a scientist, who was taught by a professional luthier. Subsequent layers were applied with $24 \mathrm{~h}$ intervals, including $8 \mathrm{~h}$ of ultraviolet (UV) irradiation. To study the impact of the individual materials and to reflect different coating systems as used for historical and modern violins, different combinations (Table 2) were investigated. In general, the applied coating materials can be separated into pretreatments (N, S and G), which penetrate the outer wood cells, and the main varnish (either A or 0 ) which forms a homogenous layer on the pretreated wood surface. In the case of the spruce plates' measurements, all possible combinations of pretreatments were studied and combined with both the alcohol and oil varnishes. For the maple plates, the different combinations of pretreatments were studied for the alcohol varnish. Besides, the influence of the oil varnish was examined on a NSG pretreatment system. As Table 2 shows, this results in eight different coating systems for the spruce plates and five different coating systems for the maple plates. For statistical analysis, each wood/coating combination was examined on five plates. The samples were weighed on a precision balance $( \pm 1 \mathrm{mg})$ after each varnishing step and at each measurement time. To enable a direct comparison between the mass changes of the spruce and maple plates, the changes were evaluated as a coating system-induced areal mass loading:

$$
\text { Areal loading }=\frac{m-m_{\text {initial }}}{b l}
$$

where $m$ is the mass, $b$ the width and $l$ the length of the plate. The subscript initial refers to the initial, unvarnished plate. The plates' thicknesses, which were considered in the density and numerical calculations, were determined during the varnish application as an average of three measurements per plate with a micrometer $( \pm 1 \mu \mathrm{m})$, and considered as constant during drying and aging.

Modal analysis: To eliminate any influences of temperature and relative humidity $(\mathrm{RH})$ changes, all wooden plates were preconditioned, varnished and measured in the same climatic chamber $\left(20^{\circ} \mathrm{C}\right.$ and $65 \%$ $\mathrm{RH})$. The general setup and process of the measurements are shown in Figure 2. The vibration was excited contact free with a sine sweep between $150 \mathrm{~Hz}$ and $10000 \mathrm{~Hz}$, generated by a loudspeaker positioned behind the plate. The out-of-plane vibration velocity frequency response function (FRF) was measured with a PSV-500 scanning laser vibrometer (Polytec $\mathrm{GmbH}$, Waldbronn, Germany) using the $\mathrm{H} 1$ estimator. This non-intrusive approach is of great advantage with regard to potential investigations on valuable violins. The measurements were conducted
Table 2: Term and composition of the studied coating systems ( $\checkmark$ present, $\boldsymbol{X}$ not present).

\begin{tabular}{|c|c|c|c|c|c|}
\hline Term & $\mathrm{N}^{\mathrm{a}}$ & $\mathbf{S}^{\mathrm{b}} \mathbf{G}^{\mathrm{c}}$ & $\begin{array}{l}\text { Varnish } \\
\left(A^{\mathrm{d}} \text { or } 0^{\mathrm{e}}\right)\end{array}$ & $\begin{array}{r}\text { No. of spruce } \\
\text { samples }\end{array}$ & $\begin{array}{r}\text { No. of maple } \\
\text { samples }\end{array}$ \\
\hline Reference & $x$ & $x x$ & $x$ & 10 & 5 \\
\hline NA & $\checkmark$ & $x x$ & $\checkmark(\mathrm{A})$ & 5 & 5 \\
\hline NO & $\checkmark$ & $x x$ & $\checkmark(0)$ & 5 & - \\
\hline NSA & $\checkmark$ & $\sqrt{x}$ & $\checkmark(A)$ & 5 & 5 \\
\hline NSO & $\checkmark$ & $\checkmark x$ & $\checkmark(0)$ & 5 & - \\
\hline NGA & $\checkmark$ & $x \checkmark$ & $\checkmark(\mathrm{A})$ & 5 & 5 \\
\hline NGO & $\checkmark$ & $x \checkmark$ & $\checkmark(0)$ & 5 & - \\
\hline NSGA & $\checkmark$ & $\checkmark \checkmark$ & $\checkmark(A)$ & 5 & 5 \\
\hline NSGO & $\checkmark$ & $\checkmark \checkmark$ & $\checkmark(0)$ & 5 & 3 \\
\hline
\end{tabular}

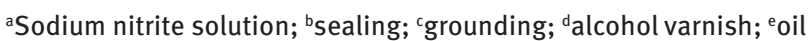
varnish.

at several equidistant points along one short and one long edge of the plate whereby the mode shapes were clearly identifiable (Figure $2 b$ ). The eigenfrequencies and their modal damping ratios were calculated by a modified method of linear prediction (Tufts and Kumaresan 1982; Lebedev 2002), which was applied for wooden beams in Van Damme et al. (2017). The procedure is based on fitting a sum of exponentially damped sine waves to the experimentally determined response. Figure 2 exemplarily shows a measured FRF (blue curve) and the calculated linear prediction (red curve) for a spruce (Figure 2d) and a maple (Figure 2e) plate measurement. The labeling of the eigenmodes refers to the number of node lines, i.e. the blue lines in Figure 2, along the short and the long edge of the plates (no. of node lines along the short edge, no. of node lines along the long edge). Due to the material properties and the dimension of the plates, different eigenmodes can fall on the same eigenfrequency, making it difficult to assign them individually. For complex FRFs, with many overlapping resonance peaks, the linear prediction method results in a more reliable estimation of the eigenfrequency and modal damping ratios than the straightforward peak identification (Remillieux et al. 2015). In Figure 2, the eigenmodes that were clearly recognizable for all measurements and that were subsequently used for further analysis are displayed and highlighted in the FRFs.

In the case of small damping values $(\xi<0.07)$, the determined modal damping ratios $\xi$ can be converted to other common damping measures according to Graesser and Wong (1992):

$$
\zeta=\frac{\tan (\delta)}{2}=\frac{\eta}{2}=\frac{Q^{-1}}{2}=\frac{\Lambda}{2 \pi}
$$

where $\tan (\delta)$ is the loss tangent, $\eta$ the loss factor, $Q$ the quality factor and $\Lambda$ the logarithmic decrement. The modal damping ratios of the fundamental bending modes, i.e. mode $(1,1),(2,0)$ and $(0,2)$, can directly be transferred to the directional properties $[(1,1)=\mathrm{LR}$, $(2,0)=\mathrm{R},(0,2)=\mathrm{L}]$. The modal damping ratio of the second torsional bending mode, however, also strongly depends on the longitudinal damping properties (McIntyre and Woodhouse 1988).

Inverse mechanical property determination process: Wood, owing to its different properties in the longitudinal, radial and tangential directions, is commonly modeled as an orthotropic material with nine elastic constants and a physical parameter, the density. Based on an iterative optimization process, by minimizing the difference between the results obtained experimentally and by a numerical model of the plate vibrations, it is possible to determine the elastic properties of the 


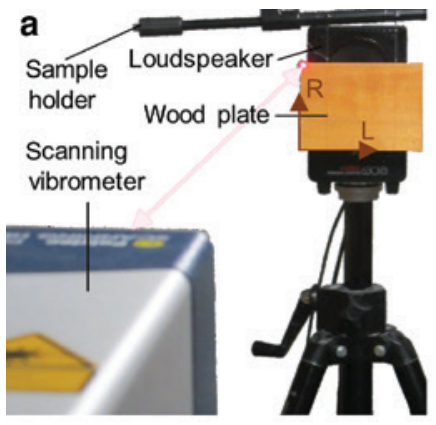

d

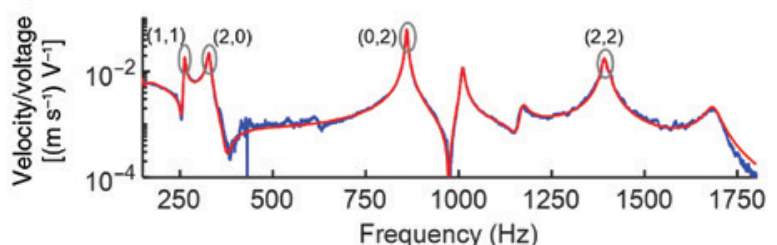

b

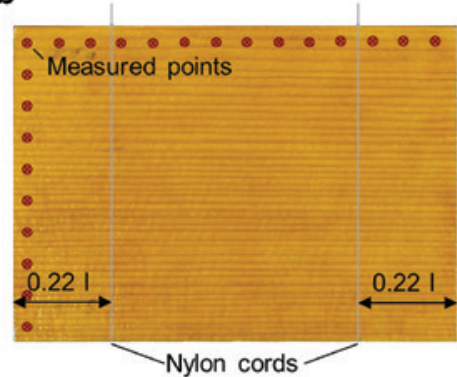

C
$(1,1)$

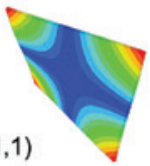

$(0,2)$

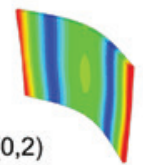

$(2,0)$

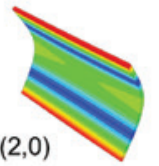

$(2,2)$

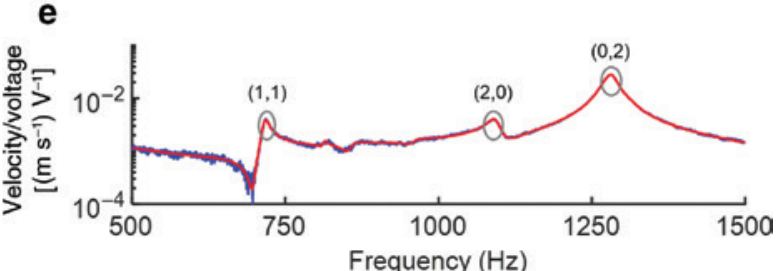

Figure 2: General setup and process of measurements for modal analysis.

(a) Setup with a scanning laser vibrometer, a plate and the loudspeaker positioned behind the plate, (b) the measured points aligned along one short and one long side shown on a spruce plate, (c) the identified mode shapes corresponding to the measured (blue) and fitted (red) frequency response functions of a (d) spruce and a (e) maple plate measurement.

plates. In the case of thin rectangular wooden plates, this approach allows identifying the in-plane stiffness properties, i.e. $E_{L}, E_{R}$ and $G_{L R}$ (McIntyre and Woodhouse 1988; Larsson 1997; Pérez Martínez et al. 2011). Within the optimization, these design variables were determined by minimizing the objective function:

$$
g=\sum_{i} \sqrt{w_{i}\left(\frac{f_{i, \text { num }}-f_{i, \text { exp }}}{f_{i, \text { exp }}}\right)^{2}}
$$

where $i$ indicates the modes considered for the analysis [i.e. $(1,1)$, $(2,0),(0,2)$ and $(2,2)], f$ the corresponding eigenfrequency determined numerically and experimentally, respectively, and $w$ a weighting factor. As initial presumption, the parameters were estimated by a closed-form eigenvalue solution for a free-free beam (Larsson 1997):

$$
\begin{aligned}
& E_{L}=f_{0,2}^{2} \rho \frac{19 l^{4}}{2 \pi^{2} h^{2}} \\
& E_{R}=f_{2,0}^{2} \rho \frac{19 b^{4}}{2 \pi^{2} h^{2}} \\
& G_{L R}=f_{1,1}^{2} \rho\left(\frac{b l}{h}\right)^{2}
\end{aligned}
$$

where $\rho$ is the homogenized density, $l$ the length, $b$ the width and $h$ the thickness of the plates. For the remaining elastic constants, common values were taken from the literature (Kretschmann 2010). As apparent from equations (4)-(6) and from sensitivity analyses (Larsson 1997; Pérez Martínez et al. 2011), the mechanical properties strongly correlate with certain eigenfrequencies. In the case of rectangular free plates, the eigenfrequencies are generally calculated with approximations obtained by the Rayleigh's method, the Rayleigh-Ritz technique or using finite element method (FEM) (Hwang and Chang 2000). For the study of the varnished plates, the density is averaged and assumed to be homogenous. The elastic moduli were calculated with the different dimensions of the spruce and maple plates in the numerical model. A preliminary comparison of the results obtained with an FEM model to a calculation based on the Rayleigh-Ritz method using the xyz algorithm (with $n=12$ ) (Visscher et al. 1991) was conducted. As for the first 18 eigenfrequencies, the relative changes between these two methods remained below $0.5 \%$, the calculation of the vibrational response for the optimization problem was performed with the computationally less expensive xyz algorithm. The optimization was conducted in MATLAB using the Nelder-Mead simplex algorithm [fminsearch (The MathWorks 1984-2017)].

Test sequence, statistical evaluation and error quantification: All spruce and maple plates were measured in their unvarnished state and subsequently after each varnishing step. This resulted in four measurements during the varnishing process: (i) initial (unvarnished), (ii) after the sodium nitrite solution application, (iii) after the remaining pretreatment application (either S, G or S and G), and (iv) after the application of alcohol or oil varnish. Moreover, drying and aging of the coating systems was studied by additional measurements that were conducted after (v) 1 month, (vi) 3 months and (vii) 6 months, and for the spruce plates additionally after (viii) 12 months and (ix) 18 months. Generally, the impact of the freshly applied layers differs from the varnish-induced changes occurring over time and are therefore considered separately. The significance of different factors (e.g. pretreatment system, alcohol or oil varnish, time evolution, spruce or maple) on the measured and calculated properties was determined using a one-way analysis of variance (ANOVA) at the 5\% significance level in MATLAB.

\section{Results and discussion}

\section{Areal loading}

Figure 3 shows the mean areal mass loadings that resulted from the varnish application during the varnishing process and over time. The final loadings varied between $62 \mathrm{~g} \mathrm{~m}^{-2}$ 
for an NA sample and $173 \mathrm{~g} \mathrm{~m}^{-2}$ in the case of spruce and $129 \mathrm{~g} \mathrm{~m}^{-2}$ in the case of maple for an NSGO plate. A direct comparison between the same coating systems on spruce (Figure 3a) and maple (Figure 3b) showed that with the exception of NA, the areal mass loading for spruce was significantly higher than that for maple. When comparing the alcohol (A) and oil varnishes (O), the oil varnish always resulted in a significantly higher loading. Considering the pretreatments, NSG showed significantly higher and $\mathrm{N}$ significantly lower loadings compared to the remaining systems at the end (after 18 and 6 months, respectively). Between NS and NG, the only significant difference occurred in combination with the alcohol varnish for the spruce plates. Moreover, the results showed that the pretreatments had an influence on the final amount of alcohol and oil varnish applied: the total areal loading of alcohol or oil varnish in combination with NSG was higher than the sum of combining NG and NS and deducting $\mathrm{N}$ (e.g. $\mathrm{NSGO}>\mathrm{NSO}+\mathrm{NSG}-\mathrm{NO}$ ). This can be explained by the sealing function of the pretreatments, which avoided an unrestricted alcohol or oil varnish penetration as for the samples treated only with $\mathrm{N}$. Therefore, in the case of manual application, more alcohol and oil varnish was present on samples without $S$ and $G$, especially in the case of the oil varnish with a slowly advancing drying process and thus more time for varnish penetration. Over time, during drying and aging of the coating systems (after 1 month), the areal loadings did not change significantly.

\section{Inverse mechanical property determination process}

The initial optimization was performed with equal weights $\mathrm{w}_{\mathrm{i}}=1$ for all modes. In the case of the spruce plates, the starting and optimized values of $E_{L}$ and $E_{R}$ differed less than $1.4 \%$ and $0.2 \%$, respectively. Therefore, the optimization problem was reduced to an optimization of $G_{L R}$. From the identified modes, $G_{L R}$ depends mainly on the first and second torsional bending modes $(1,1)$ and $(2,2)$. Of these two modes, $(2,2)$ is not overlapping with neighboring modes, as observed for $(1,1)$ (Figure 2). Therefore, the eigenfrequency and modal damping ratio determination is more precise and reliable for $(2,2)$. Accordingly, the changes in $G_{L R}$ showed similar median changes but less variation, when $(2,2)$ is weighted higher than $(1,1)([\mathrm{w}(1,1)$

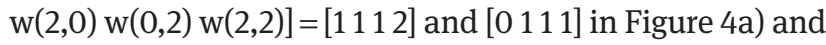
thus lead to statistically more meaningful results. Hence, the influence of $(2,2)$ was weighted twice within the optimization problem for the spruce plates $([\mathrm{w}(1,1) \mathrm{w}(2,0) \mathrm{w}(0,2)$ $\mathrm{w}(2,2)]=[1112])$. The choice of weighting factor also shows an influence on the absolute value of $G_{L R}$. Figure $4 \mathrm{~b}$ shows the distribution of $\mathrm{G}_{\mathrm{LR}}$ for all spruce plates before varnishing and after 18 months. In the case of higher weighting of $(2,2)$, i.e. fitting more precisely the higher frequency mode $(2,2)$, higher absolute values for $\mathrm{G}_{\mathrm{LR}}$ were obtained ([w $(1,1)$ $\mathrm{w}(2,0) \mathrm{w}(0,2) \mathrm{w}(2,2)]=\left[\begin{array}{llll}1 & 1 & 1 & 2\end{array}\right]$ and $\left[\begin{array}{llll}0 & 1 & 1 & 1\end{array}\right]$ in Figure 4b). This suggests that, similar to $E_{L}$ (Ouis 2002), $G_{L R}$ increases with frequency and thus that the method is also suitable to investigate frequency dependencies of the elastic moduli.

The averaged resulting moduli and modal damping ratios for the unvarnished spruce and maple plates are summarized in Table 3. Most initial properties were in the range of typically observed values and anisotropy ratios (cf. Brémaud et al. 2011). The modal damping ratio in the radial direction of spruce, however, was significantly higher than the previous measurements in the literature (cf. Haines 1979; Hutchins 1991; Sedighi Gilani et al. 2016). The values were probably estimated higher due to the close presence of a second vibration mode. The detailed data for all a

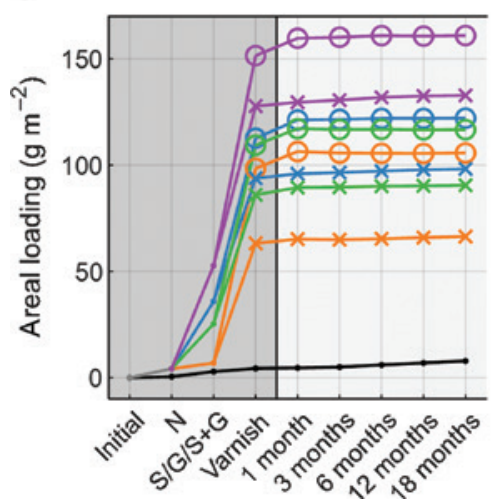

\section{b}

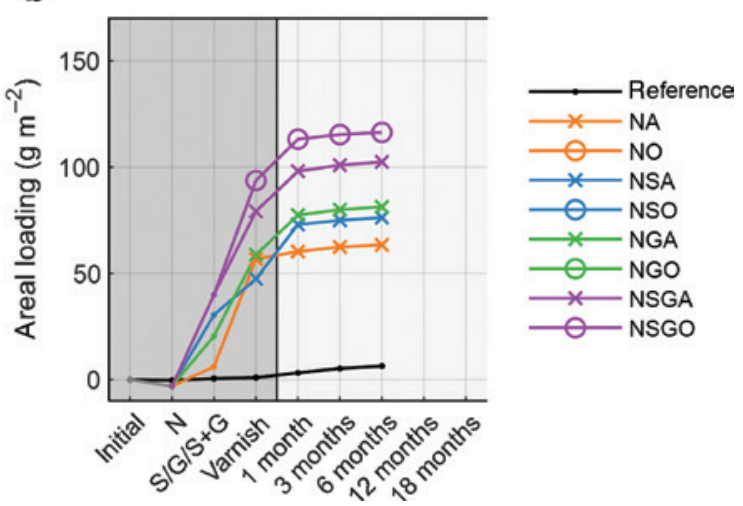

Figure 3: Mean areal mass loadings induced by the coating systems for (a) spruce plates and (b) maple plates. The slight loading increases during drying and aging resulted from an increased weight of the wood itself (see changes for reference samples), which might be explained by a potential additional moisture uptake for the aged wood. 

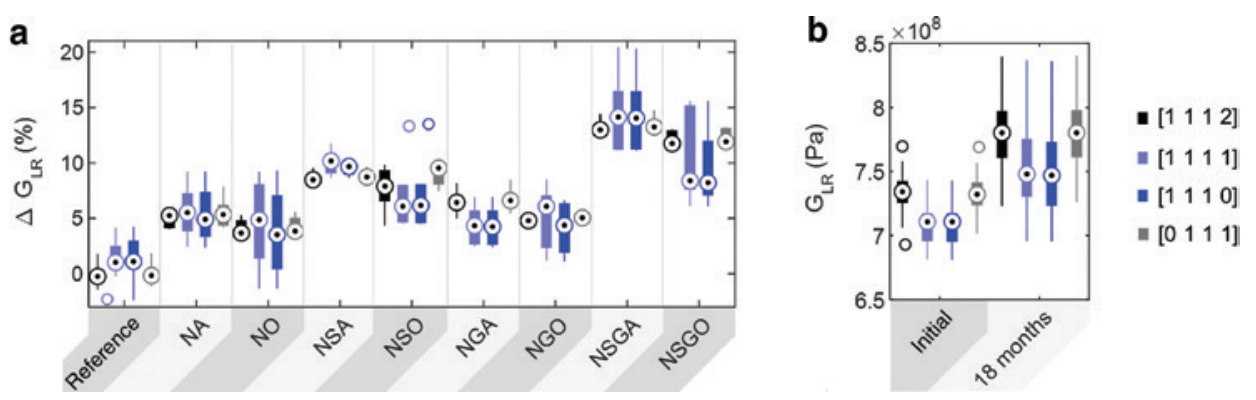

Figure 4: Influence of varnishing and the weighting factors of the eigenmodes $\left[w_{(1,1)} w_{(2,0)} w_{(0,2)} w_{(2,2)}\right]$ on the shear modulus $G_{L R}$. Box plots showing the influence of the weighting factors on $G_{L R}$ of the spruce plates for (a) different coating systems induced changes on $G_{L R}$ after 18 months and for (b) absolute values of $G_{L R}$ (of all plates) before the varnish application and after 18 months.

Table 3: Longitudinal and radial E-moduli, shear modulus and modal damping ratios of the plates before varnishing.

\begin{tabular}{lrrrrrr}
\hline & $\mathrm{E}_{\mathrm{L}}(\mathrm{GPa})$ & $\mathrm{E}_{\mathrm{R}}(\mathrm{GPa})$ & $\mathrm{G}_{\mathrm{LR}}(\mathrm{GPa})$ & $\zeta_{(0,2)}=\zeta_{\mathrm{L}}(\%)$ & $\zeta_{(2,0)}=\zeta_{\mathrm{R}}(\%)$ & $\zeta_{(1,1)} / \zeta_{(2,2)}(\%)$ \\
\hline Spruce plates $\emptyset(\mathrm{std})$ & $14.77( \pm 0.19)$ & $0.60( \pm 0.12)$ & $0.734( \pm 0.014)$ & $0.30( \pm 0.02)$ & $1.6( \pm 0.4)$ & $0.54^{\mathrm{a}}( \pm 0.03)$ \\
Maple plates $\emptyset(\mathrm{std})$ & $12.5( \pm 0.4)$ & $1.55( \pm 0.10)$ & $1.58( \pm 0.07)$ & $0.65( \pm 0.08)$ & - & $0.81^{\mathrm{b}}( \pm 0.05)$ \\
\hline
\end{tabular}

The modal damping ratios correspond to the values obtained for the identified longitudinal, radial and torsional bending modes. The standard errors of measurement (SEM) and their influence on the elastic moduli are reported in Supplementary material S3. Std, standard deviation. ${ }^{a}$ For mode $(2,2)$; bfor mode $(1,1)$.

individual samples and measurements (including the input parameters, i.e. the dimensions, masses and eigenfrequencies) are provided in Supplementary materials 1 and 2.

\section{Results for spruce plates}

The results for the mean relative changes in eigenfrequencies, elastic moduli and modal damping ratios $(\zeta)$ for the coating systems with the alcohol varnish are shown in Figure 5.

Generally, the initial influence of the coating systems differs between the longitudinal, radial and torsional modes. Regarding the eigenfrequencies and elastic moduli, the freshly applied coating systems resulted in a significant increase for the radial bending mode, whereas for the torsional (except NA and NGA) and longitudinal modes, the properties were reduced. With regard to the modal damping ratios, the freshly applied coatings resulted in a strong increase, which was most pronounced in the radial direction and for NSGA-coated samples.

The trends for the further changes occurring over time during the drying and aging of the coating systems were rather similar. Proceeding from the finished coatings, all eigenfrequencies and elastic moduli were increased. As meanwhile the mass was not subjected to significant changes, as described earlier, these increases indicate a stiffening of the coating systems over time. After 18 months, the changes in eigenfrequencies and elastic moduli of all coating systems were significantly different compared to the changes occurring for the reference plates, showing increased radial and torsional and slightly decreased longitudinal properties. For NSGA, these changes were strongest and for NA they were less noticeable. The differences observed between the different coating systems and the different wood directions diminished over time. Moreover, the large damping increases measured for freshly applied coating systems were less pronounced with time. However, in the case of the longitudinal and torsional modes, the general increase remained.

When comparing the development of the changes over time, an influence of the solvents is evident. For the NA samples, with ethanol as the main solvent, most changes happened within the first month. For the NSGA samples, with two pretreatment layers based on turpentine (S) and linseed oil $(G)$, the dynamics of the changes were slower and still apparent after 18 months. This finding is in good agreement with previous studies that also showed more rapid changes for alcohol varnishes than for (siccative-) oil-based varnishes (Barlow and Woodhouse 1990; Minato et al. 1995; Brémaud et al. 2016). Schleske (Schleske 1998), on the other hand, only noticed this effect for simple varnishes, consisting of one resin dissolved in a solvent. For more complex varnishes, containing multiple ingredients, he did not observe an influence of the solvent on the drying dynamics (Schleske 1998). 

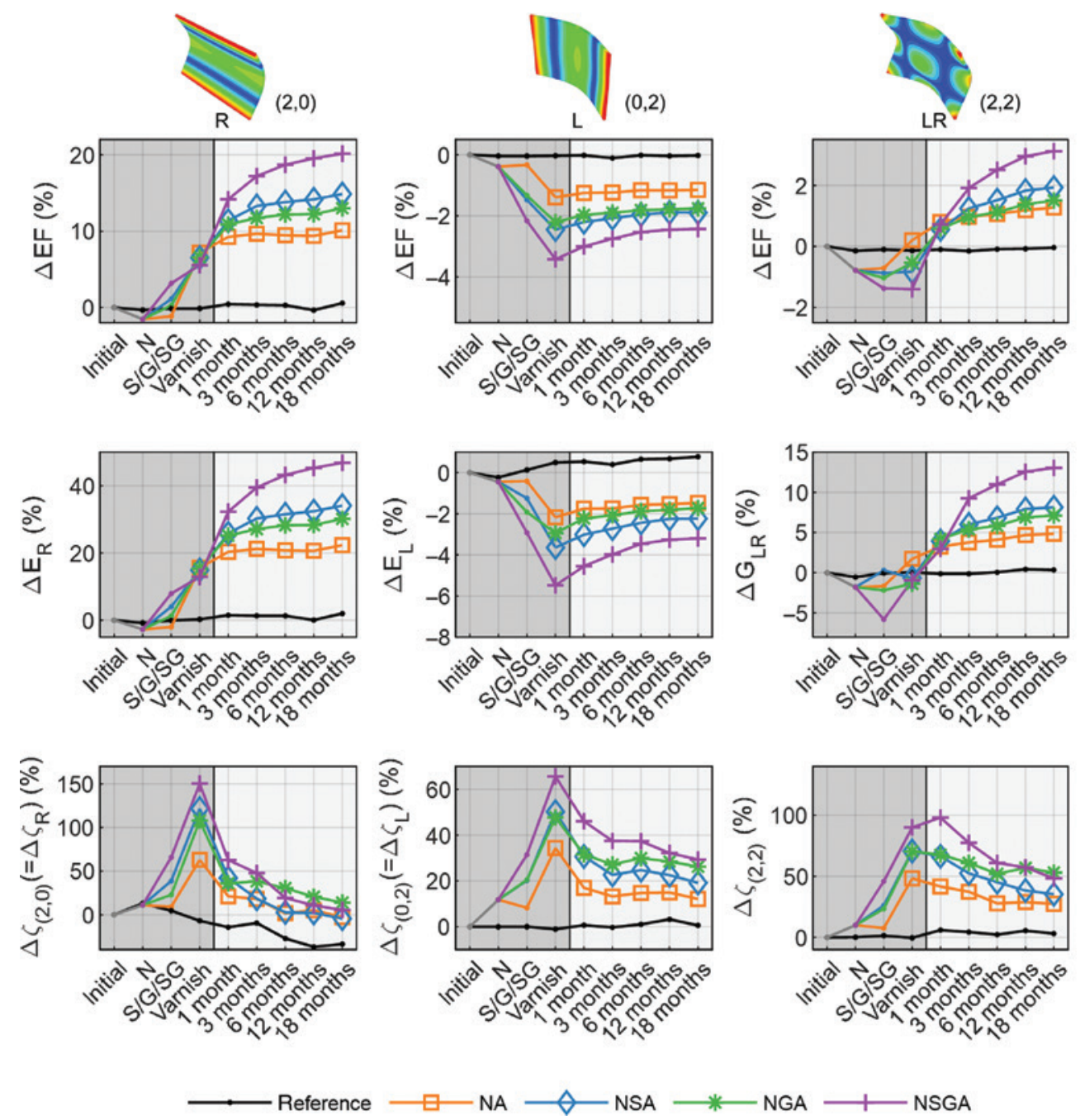

Figure 5: Mean relative changes for (left) mode $(2,0)$, (middle) mode $(0,2)$, (right) mode $(2,2)$ of the corresponding (top) eigenfrequencies, (middle) elastic moduli and (bottom) modal damping ratios for the coating systems containing alcohol varnish (A) applied to spruce plates.

To allow for comparison of the oil and alcohol varnishes, the mean relative changes for all oil varnishcontaining systems are shown in blue in Figure 6. The general trends and pretreatment influences were similar to the alcohol varnish systems (indicated in gray in Figure 6). However, some differences are obvious: the final increases for the radial and torsional eigenfrequencies and elastic moduli were smaller for the oil than for the alcohol varnish, whereas the reductions for the longitudinal properties were more pronounced. Moreover, the modal damping ratio increases were more distinct for the oil than for the alcohol varnish systems. A direct comparison between the alcohol and oil varnishes for the coatings with identical pretreatment systems showed that these differences are significant for the longitudinal and torsional modes. The findings prompt that the oil varnish has a lower E-modulus but a higher $\tan (\delta)$ than the alcohol varnish.

As for both varnishes, the impact was increased with the number of pretreatment layers applied, a dependency of the investigated parameters on the mass changes can be presumed. Figure 7 shows the relative changes in eigenfrequencies, elastic moduli and modal damping ratios after 18 months as a function of the corresponding relative mass change. Obviously, there is a correlation to the mass changes, but also a strong dependency on the type of varnish materials applied. The blue and brown ellipses illustrate the influence of the applied varnish (alcohol or oil varnish). While the results for the alcohol and oil varnishes for the longitudinal bending mode fall roughly on one line, the results for the radial bending mode and especially for the torsional bending 
$(2,0)$
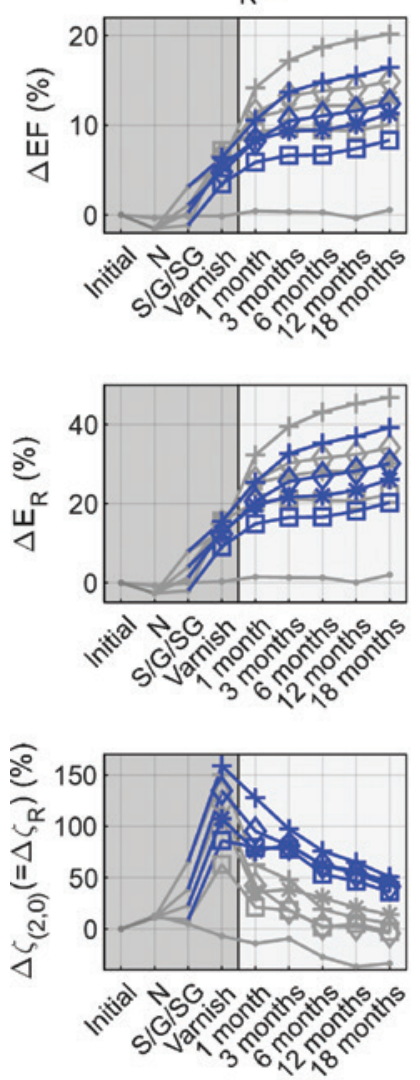
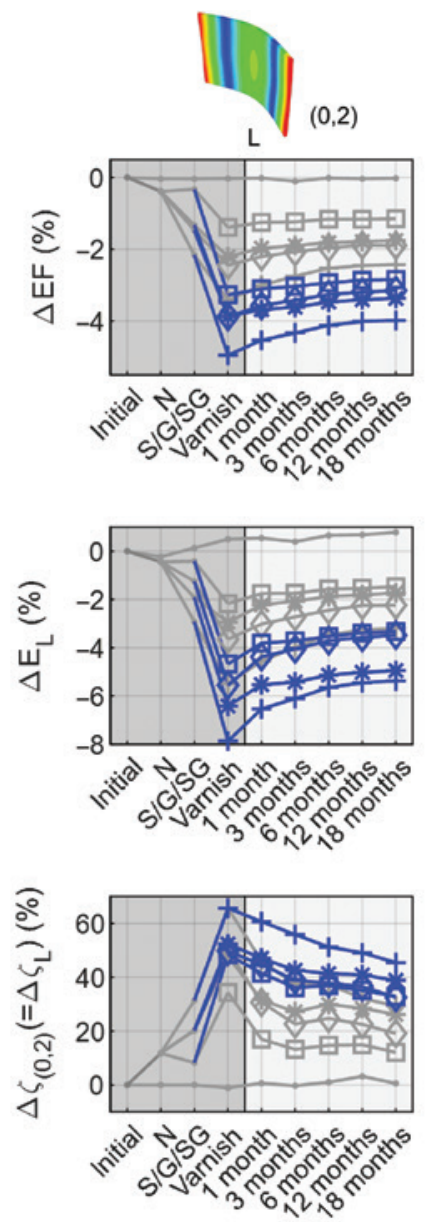

$(2,2)$
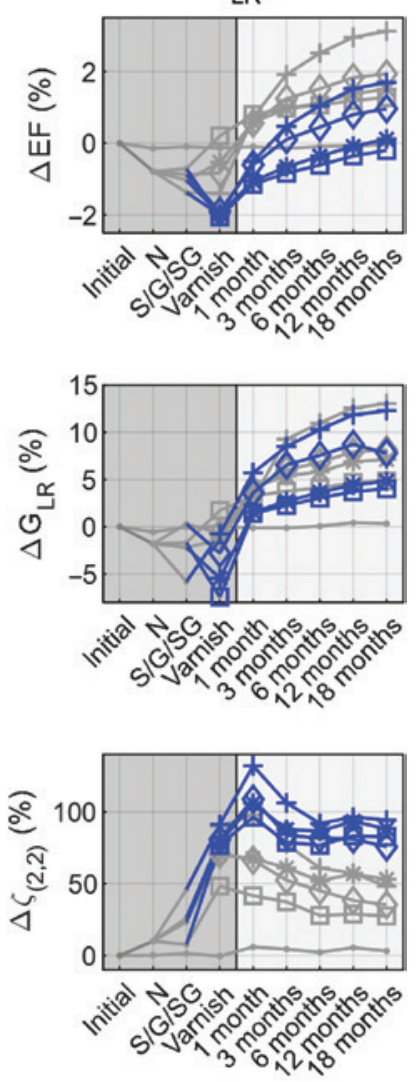
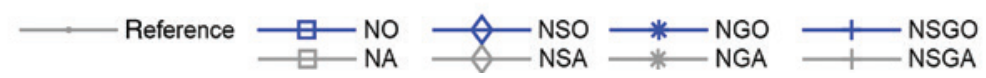

Figure 6: Mean relative changes for (left) mode $(2,0)$, (middle) mode $(0,2)$, (right) mode $(2,2)$ of the corresponding (top) eigenfrequencies, (middle) elastic moduli and (bottom) modal damping ratios for all coating systems applied on spruce plates.

mode clearly indicated the influence of the varnish, and thus its mechanical properties.

Overall, the measured changes for the radial and longitudinal properties showed similar results as previous studies on wood strips (Hutchins 1991; Minato et al. 1995; Schleske 1998; Sedighi Gilani et al. 2016; Lämmlein et al. 2019), i.e. the highest impact was recorded for the damping ratio (increase) and, in general, for the properties in the radial direction. These conclusions hold for all studied varnish materials. Even though the material properties changed less with the passage of time, they did not reach constant conditions after 18 months. The results reflect the complexity of the impact of the multi-layered coating systems and are dependent on the type of varnish materials and their mechanical properties as well as on the amount applied and the age of the coating system.
Moreover, the underlying wood properties also come into play, as shown with the following results for the varnish impact on maple wood that is commonly used for the construction of the violin back plate.

\section{Results for maple plates}

The mean changes for the different coating systems applied to the maple plates are shown in Figure 8. As the response of the radial eigenmode $(2,0)$ is overlapped by the strong longitudinal bending mode $(0,2)$ (see Figure 2 ), it was not possible to determine the precise radial modal damping ratio values.

In general, the changes in eigenfrequency and elastic moduli were less pronounced compared to spruce. 

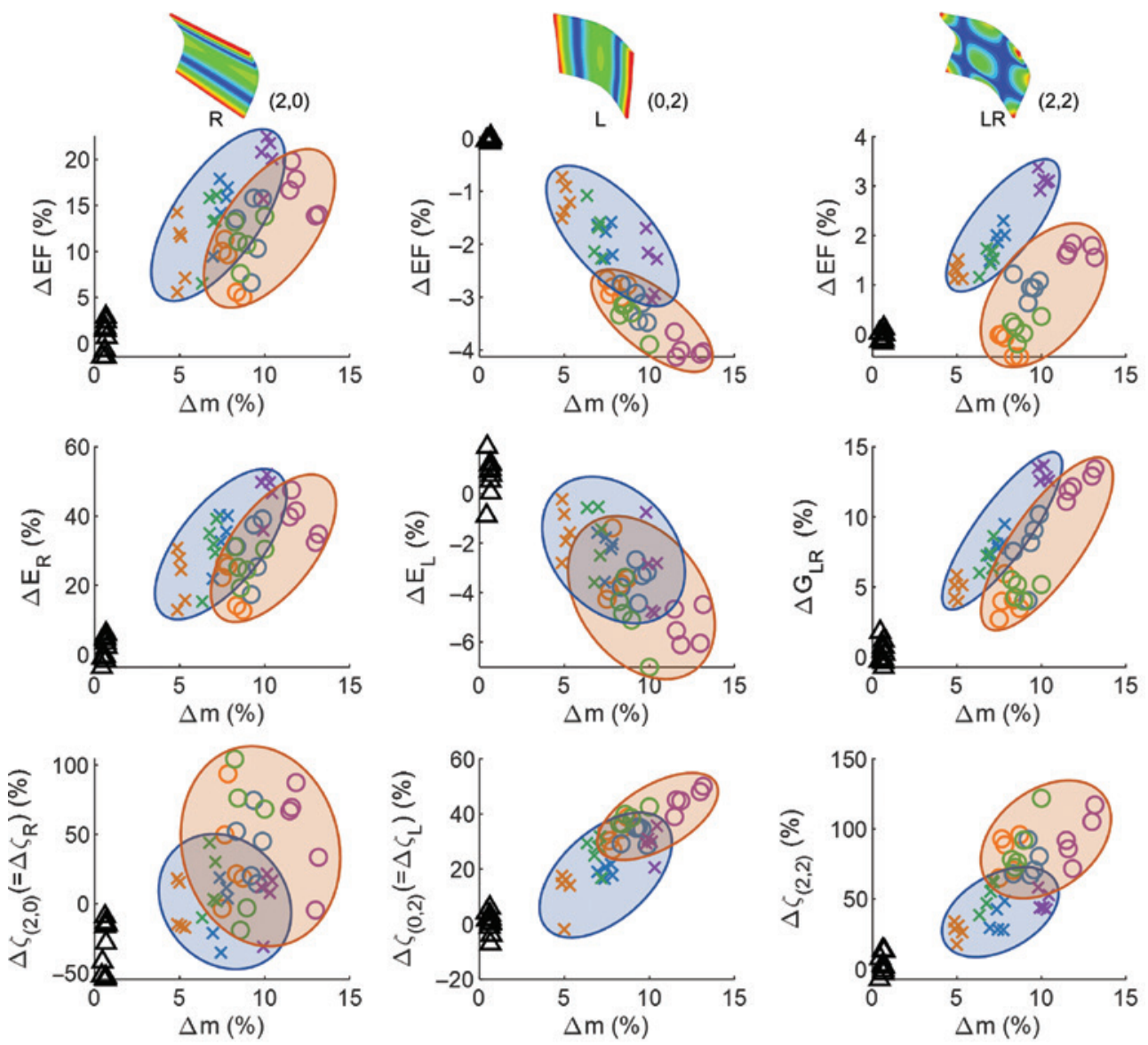

$\triangle$ Reference $\times \mathrm{NA} \times \mathrm{NSA} \times \mathrm{NGA} \times \mathrm{NSGA}$

$O$ NO O NSO O NGO O NSGO

Figure 7: Relative changes in eigenfrequency, elastic moduli and modal damping ratios as a function of $\Delta \mathrm{m}$ for the measurement after 18 months. All $A$ and $O$ varnish containing coating systems are consolidated in the blue and brown ellipses, respectively.

The results for the radial eigenfrequency and $E_{R}$ had a similar trend as the torsional changes of the spruce plates: the freshly applied varnishes resulted in a decrease, but over time, the radial eigenfrequency and $\mathrm{E}_{\mathrm{R}}$ showed an increase. This change in behavior over time could explain the contradictory results of previous studies, which observed a decrease of $E_{R}$ for maple (Haines 1980) and an increase in eigenfrequency for mulberry (Brémaud et al. 2016). In the longitudinal direction, the alcohol varnishcontaining systems resulted in an increase in eigenfrequency after 6 months, whereas the corresponding $E_{L}$ was only significantly changed for NSGA. Regarding the torsional eigenfrequency and $G_{L R}$, the only significant change was measured for the NSGA system.

The modal damping ratios showed a strong and significant increase [with an exception of the NA system for mode $(1,1)]$, as also previously reported for wood strips (Meinel 1957; Haines 1980; Eichelberger 2006). Unlike the spruce plates, the increase for the longitudinal mode was higher than that for the torsional mode.

The lower influence of the coating systems on the stiffness properties for maple than for spruce plates might be explained by the higher density of maple wood (Table 1). Moreover, the areal mass loadings of the coating systems were higher for spruce than for maple wood. Thus, the changes in mass and density were more pronounced for the spruce plates resulting in an overall higher impact. In addition, the initial wood properties also differ. The spruce plates showed a higher anisotropy than the maple plates with a higher $\mathrm{E}_{\mathrm{L}}$ but lower $\mathrm{E}_{\mathrm{R}}$-modulus (Table 3). For common mechanical varnish properties [around $\mathrm{E}=1-4 \mathrm{GPa}$ for shellac varnish, copal varnish, linseed oil, lacquers and nitrocellulose sealer (Ono 1993; Obataya et al. 2001; Simonnet et al. 2002)], $E_{\text {Varnish }}$ is higher than $\mathrm{E}_{\mathrm{R} \text {,wood }}$ and smaller than $\mathrm{E}_{\mathrm{L} \text {,wood }}$, thus, in both cases, closer to the maple than to the spruce properties. 

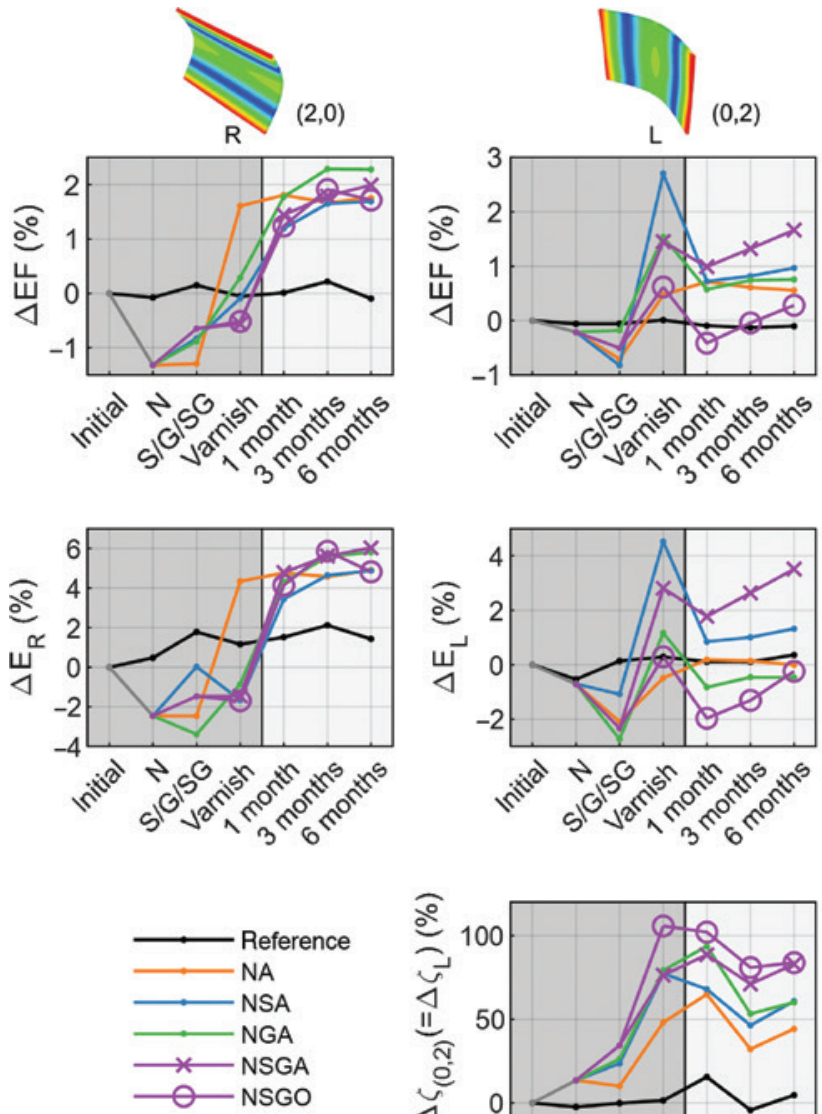
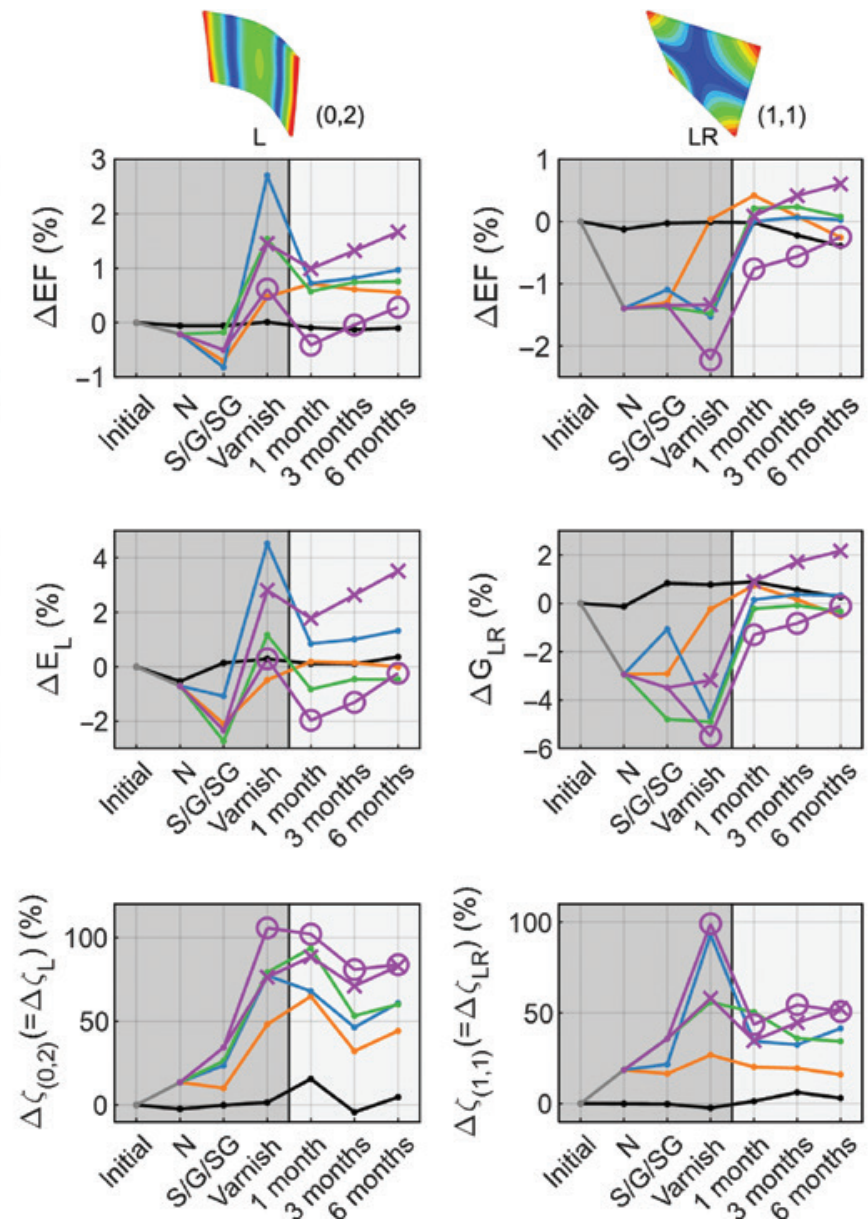

Figure 8: Mean relative changes for (left) mode $(2,0)$, (middle) mode $(0,2)$, (right) mode $(1,1)$ of the corresponding (top) eigenfrequencies, (middle) elastic moduli and (bottom) modal damping ratios for all coating systems applied to maple plates. Due to the smaller scaling of the $y$-axes, the step-wise changes over time appear less smooth than for the spruce plate measurements.

\section{Conclusions}

An interrelated analysis of the time-dependent influence of representative, traditional multi-layer and multimaterial violin coatings with a focus on their impact on the shear modulus and the elastic properties of spruce and maple was conducted. The varnish influence on the bending mode eigenfrequencies, elastic moduli and damping properties was investigated by laser vibrometer measurements. Multimodal analysis of rectangular plates was used to determine the material properties. This approach enabled a simultaneous determination of the different properties on an individual sample within one measurement. In general, the effect differed for the respective wood directions and depended on the wood and varnish material properties, the amount of varnish applied and the elapsed time span since application. The study showed that the varnishes significantly change the longitudinal, radial and shear properties, which are known to affect the instruments' vibrations and thus its sound. The modal damping ratios were strongly increased, while the eigenfrequencies and elastic moduli showed direction- and material-dependent evolutions. In contrast to the changes for spruce, the impact of varnishing on the eigenfrequencies and elastic moduli of the maple plates was relatively low.

The knowledge of the potential influence of a coating system on the vibrational properties and elastic moduli is important for luthiers, as it prevents unwanted changes. However, it should be considered that the varnish influence is also subjected to changes in time, especially during the first 3 months after treatment. Being non-invasive, the approach of performing modal analysis and a subsequent inverse mechanical material property determination is, in combination with an accurate FEM model, suitable for further investigations on the impact of coating systems on violin plates, the complete instruments or on frequency dependencies of the studied properties. Moreover, the 
method can be employed to study the influence of wood treatments or new materials used for the manufacture of instruments.

Acknowledgments: The first author thanks R. Viala and S. Cogan of FEMTO-ST, Besançon, for an introduction to the general approach and methods and the possibility to conduct a feasibility study. Further acknowledgements go to M. Sedighi Gilani for initiating the COST project, to $\mathrm{U}$. Dederer for providing and explaining her knowledge about traditional varnish materials and processes and to D. Heer for the wood sample preparation.

Research funding: The work was funded by the COST Project C15.0082.

Employment or leadership: None declared.

Honorarium: None declared.

Conflict of interest: The authors declare that they have no conflict of interest.

\section{References}

Barlow, C., Woodhouse, J. (1990) The influence of varnish on the properties of spruce plates. Proc. Ins. Ac. 2:765-770.

Brémaud, I., Gril, J., Thibaut, B. (2011) Anisotropy of wood vibrational properties: dependence on grain angle and review of literature data. Wood Sci. Technol. 45:735-754.

Brémaud, I., Karami, E., Bardet, S., Gilles, N., Perego, F., Zare, S., Gril, J. (2016) Changes in vibrational properties of coated wood through time from application of varnish, with recipes used in European or Iranian string instruments making. In: Wooden Musical Instrument Conservation and Knowledge Conference, WoodMusICK. Barcelona. pp. 93-96.

Bucur, V. (2016) The varnish. In: Handbook of Materials for String Musical Instruments. Springer International Publishing, Cham. pp. 373-453.

Cattani, G., Dunbar, R.L.M., Shapira, Z. (2013) Value creation and knowledge loss: the case of Cremonese stringed instruments. Organ. Sci. 24:813-830.

Echard, J.P., Lavedrine, B. (2008) Review on the characterisation of ancient stringed musical instruments varnishes and implementation of an analytical strategy. J. Cult. Herit. 9:420-429.

Echard, J.P., Bertrand, L., von Bohlen, A., Le Ho, A.S., Paris, C., Bellot-Gurlet, L., Soulier, B., Lattuati-Derieux, A., Thao, S., Robinet, L., Lavedrine, B., Vaiedelich, S. (2010) The nature of the extraordinary finish of Stradivari's instruments. Angew. Chem. Int. Ed. 49:197-201.

Eichelberger, K. (2006) Ermittlung von Kriterien zur Beurteilung der Lackqualität im Musikinstrumentenbau und Untersuchung von neuen Lackrezepturen. Inst. f. Musikinstrumentenbau, Techn. Univ. Dresden, Zwota. pp. 77. DOI: 10.2314/GBV:512069697.

Fiocco, G., Rovetta, T., Invernizzi, C., Albano, M., Malagodi, M., Licchelli, M., Re, A., Lo Giudice, A., Lanzafame, G.N., Zanini, F. (2019) A micro-tomographic insight into the coating systems of historical bowed string instruments. Coatings 9:81.

Graesser, E., Wong, C. (1992) The relationship of traditional damping measures for materials with high damping capacity: a review. In: STP1169-EB M3D: Mechanics and Mechanisms of Material Damping. Eds. Kinra, V., Wolfenden, A. ASTM, West Conshohocken, PA, USA. pp. 316-343. https://doi/org/10.1520/STP17969S.

Haines, D.W. (1979) On musical instrument wood. Catgut Acoust. Soc. Newsl. 31:23-32.

Haines, D. (1980) On musical instrument wood Part II. Catgut Acoust. Soc. Newsl. 33:19-23.

Holz, D. (1995) Materialuntersuchungen zum langjährigen akustischen Einfluß einer Lackierung. Musikinstrum. 6-7: 98-105.

Hutchins, M. (1991) Effects on spruce test strips of four-year application on four different sealers plus oil varnish. Catgut Acoust. Soc. J. 1:11-12.

Hwang, S.-F., Chang, C.-S. (2000) Determination of elastic constants of materials by vibration testing. Compos. Struct. 49:183-190.

Kretschmann, D. (2010) Mechanical properties of wood. In: Wood handbook: wood as an engineering material. US Dept. of Agriculture, Forest Service, Forest Products Laboratory, Madison, WI. pp. 5.1-5.46.

Lämmlein, S.L., Mannes, D., van Damme, B., Burgert, I., Schwarze, F.W.M. (2019) Influence of varnishing on the vibromechanical properties of wood used for violins. J. Mater. Sci. 54:8063-8095.

Larsson, D. (1997) Using modal analysis for estimation of anisotropic material constants. J. Eng. Mech. 123:222-229.

Lebedev, A. (2002) Method of linear prediction in the ultrasonic spectroscopy of rock. Acoust. Phys. 48:339-346.

McIntyre, M., Woodhouse, J. (1988) On measuring the elastic and damping constants of orthotropic sheet materials. Acta Metallurgica. 36:1397-1416.

Meinel, H. (1957) Regarding the sound quality of violins and a scientific basis for violin construction. J. Acoust. Soc. Am. 29:817-822.

Minato, K., Akiyama, T., Yasuda, R., Yano, H. (1995) Dependence of vibrational properties of wood on varnishing during its drying process in violin manufacturing. Holzforschung 49:222-226.

Obataya, E., Ohno, Y., Norimoto, M., Tomita, B. (2001) Effects of oriental lacquer (urushi) coating on the vibrational properties of wood used for the soundboards of musical instruments. Acoust. Sci. Technol. 22:27-34.

Ono, T. (1993) Effects of varnishing on acoustical characteristics of wood used for musical instrument soundboards. J. Acoust. Soc. Jpn. (E). 14:397-407.

Ouis, D. (2002) On the frequency dependence of the modulus of elasticity of wood. Wood Sci. Technol. 36:335-346.

Padding, K. (2005) A rational look at the classical Italian coatings. J. Violin Soc. Am.: VSA Papers. 1:11-25.

Pérez Martínez, M.A., Poletti, P., Gil Espert, L. (2011) Vibration testing for the evaluation of the effects of moisture content on the in-plane elastic constants of wood used in musical instruments. In: Vibration and Structural Acoustics Analysis: Current Research and Related Technologies. Eds. 
Vasques, C.M.A., Dias Rodrigues, J. Springer Netherlands, Dordrecht. pp. 21-57.

Remillieux, M.C., Ulrich, T.J., Payan, C., Rivière, J., Lake, C.R., Le Bas, P.-Y. (2015) Resonant ultrasound spectroscopy for materials with high damping and samples of arbitrary geometry. J. Geophys. Res-Sol. Ea. 120:4898-4916.

Rodgers, O.E. (1988) The effect of the elements of wood stiffness on violin plate vibration. J. Catgut. Acoust. Soc. 1:2-8.

Schelleng, J.C. (1968) Acoustical effects of violin varnish. J. Acoust. Soc. Am. 44:1175-1183.

Schleske, M. (1998) On the acoustical properties of violin varnish. Catgut Acoust. Soc. J. 3:27-43.

Schleske, M. (2002) Empirical tools in contemporary violin making: Part I. Analysis of design, materials, varnish, and normal modes. Catgut Acoust. Soc. J. 4:50-64.

Sedighi Gilani, M., Pflaum, J., Hartmann, S., Kaufmann, R., Baumgartner, M., Schwarze, F.W.M.R. (2016) Relationship of vibro-mechanical properties and microstructure of wood and varnish interface in string instruments. Appl. Phys. A. 122:1-11.

Simonnet, C., Gibiat, V., Halary, J.-L. (2002) Physical and chemical properties of varnishes and their vibrational consequences. PACS Ref. 43:75.

Spinella, A., Malagodi, M., Saladino, M.L., Weththimuni, M.L., Caponetti, E., Licchelli, M. (2017) A step forward in disclosing the secret of stradivari's varnish by NMR spectroscopy. J. Polym. Sci. Pol. Chem. 55:3949-3954.

The MathWorks, I. (1984-2017) MATLAB and Statistics Toolbox Release 2018a. Natick, Massachusetts, United States.
Tufts, D.W., Kumaresan, R. (1982) Estimation of frequencies of multiple sinusoids: making linear prediction perform like maximum likelihood. Proceedings of the IEEE. 70:975-989.

Van Damme, B., Schoenwald, S., Zemp, A. (2017) Modeling the bending vibration of cross-laminated timber beams. Eur. J. Wood Wood Prod. 75:985-994.

Viala, R., Placet, V., Cogan, S., Foltête, E. (2016) Model-based effects screening of stringed instruments. In: Model Validation and Uncertainty Quantification, Volume 3. Eds. Barthorpe, R., Platz, R., Lopez, I., Moaveni, B., Papadimitriou, C. Springer International Publishing, Cham. pp. 151-157.

Viala, R., Placet, V., Cogan, S. (2018) Identification of the anisotropic elastic and damping properties of complex shape composite parts using an inverse method based on finite element model updating and 3D velocity fields measurements (FEMU-3DVF): Application to bio-based composite violin soundboards. Compos. Part A-Appl. S. 106:91-103.

Visscher, W.M., Migliori, A., Bell, T.M., Reinert, R.A. (1991) On the normal modes of free vibration of inhomogeneous and anisotropic elastic objects. J. Acoust. Soc. Am. 90:2154-2162.

Woo Yang Chung, S.H.P. (2000) Studies on the vibrational modal analysis of solid woods for making the violin - Part 2. The effects of coating materials on the resonant frequency of European spruce and maple. Korea Furnit. Soc. 11:45-52.

Supplementary Material: The online version of this article offers supplementary material (https://doi.org/10.1515/hf-2019-0182). 